\title{
Ice Condensation as a Planet Formation Mechanism
}

\author{
Katrin Ros \\ Lund Observatory, Department of Astronomy and Theoretical physics, \\ Lund University, Box 43, SE-221 00 Lund, Sweden \\ email: katrin.ros@astro.lu.se
}

\begin{abstract}
Particles in protoplanetary discs grow rapidly to millimetre-sizes via coagulation, but further growth to centimetre-sized pebbles is not yet completely understood. We investigate particle growth by ice condensation in a model where we take the dynamical behaviour of vapour and ice particles into account, as well as the size evolution due to condensation and sublimation. Our results show that efficient growth from dust to pebbles is possible close to the water ice line at $\sim 3 \mathrm{AU}$, with particles growing from millimetres to decimetres on a time scale of $10000 \mathrm{yr}$.
\end{abstract}

Keywords. Methods: numerical, planetary systems: formation, planetary systems: protoplanetary discs

\section{Introduction}

Millimetre-sized particles in protoplanetary discs form by collisions in which dust grains stick together via contact forces. For larger particles, however, experiments show that collisions tend to lead to fragmentation or bouncing rather than to sticking (Blum \& Wurm 2008). This is problematic since it is known from observations that pebbles, approximately centimetre-sized particles, do exist in protoplanetary discs (Testi et al. 2003). These pebbles are needed for continued growth towards planetesimals and planets, both for further coagulation and for clumping via streaming instabilities, in vortices or in pressure bumps (Johansen et al. 2007; Barge \& Sommeria 1995; Johansen et al. 2009). Accretion of icy pebbles is also a way to efficiently form the cores of ice giants and gas giants (Lambrechts \& Johansen 2012). We have investigated the formation of pebbles via condensation, close to the water ice line at $\sim 3 \mathrm{AU}$ (Lecar et al. 2006). Outwards of ice lines solid densities are enhanced as material condenses out, leading to larger particles sizes. Additionally, collisions between water ice particles are less destructive than corresponding ones for silicates (Wada et al. 2009). Particles outwards of the ice line can thus profit from both growth by condensation and of an enhanced coagulation probability.

\section{Model}

We have developed an idealized condensation model, in which we investigate particle size evolution due to condensation and sublimation in a dynamical model. We take into account the coupling of vapour and particles to the turbulent gas, and model the resulting turbulent diffusion as a random walk. Sedimentation to the midplane as particles decouple from the turbulent eddies is also accounted for, as well as radial drift due to the headwind particles experience from the pressure-supported gas. Condensation and sublimation are modelled in a Monte Carlo scheme, whereas particle collisions are ignored. For a more detailed description of the model, see Ros \& Johansen 2013. 


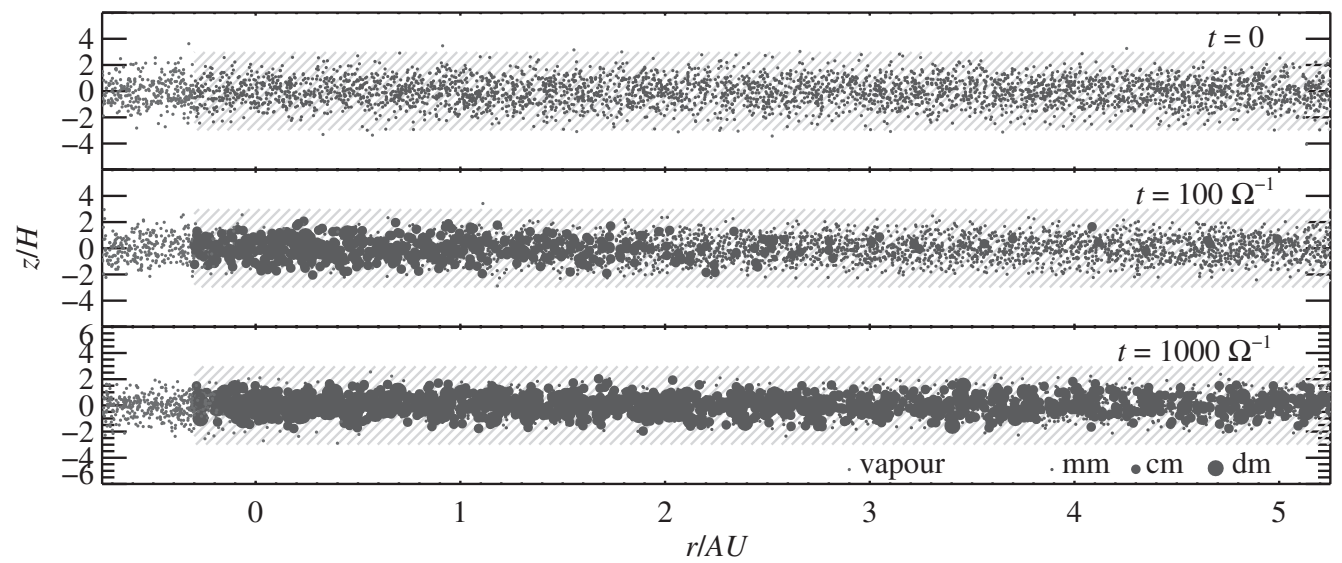

Figure 1. Distribution and sizes of particles at $t=0, t=100 \Omega^{-1}$ and $t=1000 \Omega^{-1}$, where $1 \Omega^{-1} \approx 1$ yr at $3 \mathrm{AU}$, where the ice line is located. Ice particles (shown as blue filled circles) grow and sediment to the midplane as vapour (shown as red dots) condenses onto them. The region marked by grey dashed lines is the condensation region, which consists of the cold midplane region $(|z| \lesssim 3 H$, where $H$ is scale heights) outwards of the radial ice line $(r \gtrsim 3 A U)$. The number of particles is inversely scaled with size for visibility.

\section{Conclusions}

We find that ice condensation is an efficient growth mechanism for particles up to sizes of large pebbles. In our simulations, particles grow from millimetres to decimetres on a time scale of $10000 \mathrm{yr}$ in a turbulent disc $\left(\alpha=10^{-2}\right.$, where the strength of the turbulence is parameterized by the dimensionless constant $\alpha$ introduced by Shakura \& Sunyaev 1973). These particles are large enough to explain the observed pebbles in protoplanetary discs and to enable further growth into planetesimals via instabilities and gravitational collapse. Figure 1 shows the distribution and sizes of ice particles at three different times.

We find that radial mixing due to turbulent diffusion plays an important role. Large particles form preferentially close to the radial ice line at $\sim 3 \mathrm{AU}$, but are radially transported outwards throughout the entire simulation domain. This suggests that the enhanced particle growth close to ice lines has implications for the particle size distribution also farther out in the disc.

\section{References}

Barge, P. \& Sommeria, J. 1995, A\& A, 295, L1

Blum, J. \& Wurm, G. 2008, ARAA, 46, 21

Johansen, A., Oishi, J. S., Mac Low, M.-M., et al. 2007, Nature, 448, 1022

Johansen, A., Youdin, A., \& Klahr, H. 2009, ApJ, 697, 1269

Lambrechts, M. \& Johansen, A. 2012, A\& $A, 544$, A32

Lecar, M., Podolak, M., Sasselov, D., \& Chiang, E. 2006, ApJ, 640, 1115

Ros, K. \& Johansen, A. 2013, A\& A, 552, A137

Shakura, N. I. \& Sunyaev, R. A. 1973, A\& A, 24, 337

Testi, L., Natta, A., Shepherd, D. S., \& Wilner, D. J. 2003, A\&A, 403, 323

Wada, K., Tanaka, H., Suyama, T., Kimura, H., \& Yamamoto, T. 2009, ApJ, 702, 1490 